\title{
A PROBLEM ON GROWTH SEQUENCES OF GROUPS
}

\author{
A. ERFANIAN \\ (Received 6 October 1993; revised 16 November 1993) \\ Communicated by H. Lausch
}

\begin{abstract}
The aim of this paper is to consider Problem 1 posed by Stewart and Wiegold in [6]. The main result is that if $G$ is a finitely generated perfect group having non-trivial finite images, then there exists a finite image $B$ of $G$ such that the growth sequence of $B$ is eventually faster than that of every finite image of $G$. Moreover we investigate the growth sequences of simple groups of the same order.
\end{abstract}

1991 Mathematics subject classification (Amer. Math. Soc.): $20 \mathrm{~F} 05$.

\section{Introduction}

Let $G$ be a finitely generated group, and $G^{n}$ the $n$th direct power of $G$. The growth sequence of $G$ is the sequence $\left\{d\left(G^{n}\right)\right\}$, where $d\left(G^{n}\right)$ is the minimum number of generators of $G^{n}$. Wiegold gave a very tight description on the growth sequences of finite groups in $[5,7,8,9,10]$. The rough picture is that if $G$ is perfect, the growth sequence of $G$ increases roughtly logarithmically in $n$ and if $G$ is not perfect, then $d\left(G^{n}\right)=n d\left(G / G^{\prime}\right)$ for large enough $n$. For infinite groups, the situation is less clear. If $G$ is perfect, the growth sequence of $G$ is bounded above by a logarithmic function of $n$ and if $G$ is not perfect, then again we have $d\left(G^{n}\right)=n d\left(G / G^{\prime}\right)$ for large enough $n$ (see [11]). There are several difficult problems left in the case of infinite groups; in particular, when $G$ is a finitely generated perfect group.

The present article considers Problem 1 posed by Stewart and Wiegold in [6], as follows:

ProBlem. Let $G$ be a finitely generated group having a non-trivial finite image. Is the growth sequence of $G$ eventually the same as that of a finite image of $G$ ?

(C) 1995 Australian Mathematical Society $0263-6115 / 95 \$ A 2.00+0.00$ 
This is certainly true if $G$ is not perfect. For, by the above remarks, $d\left(G^{n}\right)=$ $n d\left(G / G^{\prime}\right)$ for large $n$, in this case. However, as a finitely generated abelian group, $G / G^{\prime}$ has a finite image $X$ of prime-power order with $d\left(G / G^{\prime}\right)=d(X)$, and for all $n$, $d\left(X^{n}\right)=n d(X)=n d\left(G / G^{\prime}\right)$. In this paper, we prove that there exists a finite image $B$ of $G$ such that the growth sequence of $B$ is eventually as large as that of every finite image of $G$. This question was left undecided in [6]. So the problem shortens to this: Is the growth sequence of $G$ eventually the same as that of $B$ ? We have not been able to resolve this, but we believe that the growth sequence of $G$ could be faster than $B$ in some cases. We prove the following result, which is an improvement of $[6$, Theorem A].

THEOREM A'. Let $G$ be a finitely generated perfect group having non-trivial finite images. Then there is a finite image $B$ of $G$ such that the growth sequence of $B$ is eventually as large as that of every finite image of $G$; that is to say, there exists a positive integer $K$ depending only on $G$ such that for every finite image $H$ of $G$, $d\left(B^{n}\right) \geq d\left(H^{n}\right)$ for $n \geq K$.

PROOF. Suppose that $S$ is a non-trivial finite image of $G$ of the smallest order. From the classification of the finite simple groups and a theorem of Artin [1], there are up to isomorphism at most two possibilities for $S$. If $S$ is unique, then by Theorem $\mathrm{A}$ in [6] we can choose $B=S^{\lambda}$, where $S^{\lambda}$ is the hightest power of $S$ that is an image of $G$. So let us consider the case when $S$ is not unique. Suppose that $S$ and $T$ are two non-trivial images of $G$ of the smallest order, and let $S^{\lambda}$ and $T^{\mu}$ be the highest powers of $S$ and $T$ respectively that are images of $G$. Suppose that $H$ is a non-trivial finite image of $G$; let $S_{1}, S_{2}, \ldots, S_{r}$ be the simple images of $H$, and $S_{1}^{\lambda_{1}}, S_{2}^{\lambda_{2}}, \ldots, S_{r}^{\lambda_{r}}$ the highest powers of $S_{1}, S_{2}, \ldots, S_{r}$ that are images of $H$. There are three cases for $S$ and $T$, as follows:

CASE 1. Suppose that $S$ and $T$ occur among $S_{1}, S_{2}, \ldots, S_{r} ;$ say $S=S_{1}$ and $T=S_{2}$. By a result of Gaschütz [3] (See $[8,10])$,

$$
d\left(H^{n}\right)=\max \left\{d(H), d\left(S_{1}^{\lambda_{1} n}, \ldots, d\left(S_{r}^{\lambda_{r} n}\right)\right\}\right.
$$

for all $n$. By the second part of the proof of Theorem $\mathrm{A}$ in [6], there is a number $L$ depending only on $G$ such that $d\left(H^{n}\right)=\max \left\{d(H), d\left(S^{\lambda_{1} n}\right), d\left(T^{\lambda_{2} n}\right)\right\}$ for all $n \geq L$. Thus $d\left(H^{n}\right)=\max \left\{d\left(S^{\lambda_{1} n}\right), d\left(T^{\lambda_{2} n}\right)\right\}$ provided $n \geq L, d\left(S^{\lambda_{1} n}\right) \geq d(G)$ and $d\left(T^{\lambda_{2} n}\right) \geq d(G)$. Since this holds whenever $\log _{s} \lambda_{1} n \geq d(G)$ and $\log _{s} \lambda_{2} n \geq d(G)$ where $s=|S|$, and also

$$
d\left(S^{\lambda n} \times T^{\mu n}\right) \geq d\left(S^{\lambda_{1} n} \times T^{\lambda_{2} n}\right) \geq d\left(H^{n}\right)
$$

by [7], we can therefore choose $B=S^{\lambda} \times T^{\mu}$ in this case. 
CASE 2. Suppose that $S$ is one of $S_{1}, S_{2}, \ldots, S_{r}$, say $S=S_{1}$, but $T$ is not. Again by the same method as in Case 1 , there is a constant $K$ depending only on $G$ such that $d\left(S^{\lambda n}\right) \geq d\left(H^{n}\right)$. It is clear that $d\left(S^{\lambda n} \times T^{\mu n}\right) \geq d\left(H^{n}\right)$, so we can choose $B=S^{\lambda} \times T^{\mu}$ here.

CASE 3. Suppose that $S$ and $T$ are not among $S_{1}, S_{2}, \ldots, S_{r}$. As in the first part of proof of Theorem A in [6], we see that $d\left(S^{\lambda n}\right), d\left(T^{\mu n}\right) \geq d\left(H^{n}\right)$ for large $n$. So there are three possibilities for $\lambda$ and $\mu$ as follows:

(i) $\lambda<\mu$ : It is clear that $d\left(S^{\lambda n}\right) \geq d\left(T^{\lambda n}\right)$ and $d\left(T^{\mu n}\right) \geq d\left(s^{\lambda n} \times T^{\lambda n}\right)$, because $T^{\lambda}$ and $S^{\lambda} \times T^{\lambda}$ are images of $G$. So we can choose $B=T^{\mu}$.

(ii) $\mu<\lambda$ : As in (i), we see that $S^{\lambda}$ works.

(iii) $\lambda=\mu$ : Here we can choose $B=S^{\lambda} \times T^{\mu}$ and the proof of Theorem $\mathrm{A}^{\prime}$ is complete.

It is possible that $S^{\lambda}$ or $T^{\lambda}$ can be chosen for $B$ in case (iii), but we have been unable to check this. Let us consider the case $\lambda=\mu=1$ as an example. Then we have two finite images $S$ and $T$ of $G$ of the smallest order (which must, of course, be simple). It follows from the classification of the finite simple groups and a theorem of Artin [1] that the possibilities for $S$ and $T$ are as follows:

(a) $S=A_{8}, T=P S L(3,4)$.

(b) $S=P S p(2 m, q), T=0^{\prime}(2 m+1, q)$ where $m \geq 3$ and $q$ is an odd prime-power.

THEOREM B. Suppose that $S=A_{8}$ and $T=P S L(3,4)$. Then $d\left(S^{n}\right) \leq d\left(T^{n}\right)$ for large enough $n$.

PROOF. For any finite group $U$, set $h(m, U)=\max \left\{n: d\left(U^{n}\right) \leq m\right\}$. By [7], we have $h(m, S)=\mid$ Aut $\left.S\right|^{-1}|S|^{m}(1-\epsilon(m))$, and $h(m, T)=\mid$ Aut $\left.T\right|^{-1}|T|^{m}(1-\eta(m))$, where $\eta(m), \epsilon(m) \rightarrow 0$ as $m \rightarrow \infty$. Thus, as $m \rightarrow \infty, h(m, S) / h(m, T) \rightarrow$ $\mid$ Aut $T|/|$ Aut $S \mid=6$ by [2]. Thus $h(m, S)>h(m, T)$ for large enough $m$ and then $d\left(S^{n}\right) \leq d\left(T^{n}\right)$ for large $n$.

COROLlaRY. For each $\lambda$ in (iii) of Case $3, S$ and $T$ as Theorem $B$, the growth sequence of $T^{\lambda}$ is faster than that of every finite image of $G$ in Theorem $A^{\prime}$.

We can say less about the second possibility for $S$ and $T$. However, the difference in the growth sequence is very small indeed:

THEOREM C. Suppose that $S=P \operatorname{Sp}(2 m, q)$ and $T=0^{\prime}(2 m+1, q)$ with $m \geq 3$, $q$ an odd prime-power. Then $\left|d\left(S^{n}\right)-d\left(T^{n}\right)\right|=0$ or 1 for large enough $n$.

Proof. We know that $\mid$ Aut $S|=|$ Aut $T \mid$ by Liebeck, Praeger and Saxl [4]. Set $s=|S|$ and $a=\mid$ Aut $S \mid$. We have (see [7]) for sufficiently large $n$,

$$
\log _{s} n+\log _{s} a<d\left(S^{n}\right), d\left(T^{n}\right) \leq \log _{s} n+\log _{s} a+1+\varphi(n)
$$


where $\varphi(n) \rightarrow 0$ as $n \rightarrow \infty$. In considering upper and lower bounds, two cases arise.

(1) $\log _{s} n+\log _{s} a$ is an integer. For large $n$, it is clear that $d\left(S^{n}\right)=d\left(T^{n}\right)=$ $\log _{s} n+\log _{s} a+1$.

(2) $\log _{s} n+\log _{s} a$ is not an integer. We see easily that for large $n, d\left(S^{n}\right)$ and $d\left(T^{n}\right)$ are both one of the two smallest integers greater than $\log _{s} n+\log _{s} a$. Thus $\left|d\left(S^{n}\right)-d\left(T^{n}\right)\right| \leq 1$ and the proof of the theorem is complete.

The author wishes to acknowledge with thanks the valuable help of Professor $\mathrm{J}$. Wiegold, under whose supervision the work was done.

\section{References}

[1] E. Artin, 'The orders of the classical simple groups', Comm. Pure Appl. Math. 8 (1955), 455-472.

[2] M. Aschbacher, 'On the maximal subgroups of the finite classical groups', Invent. Math. 76 (1984), 469-514.

[3] W. Gaschütz, 'Zu einem von B. H. and H. Neumann gestellten problem', Math. Nachr. 14 (1955), 249-252.

[4] M. W. Liebeck, C. E. Praeger and J. Saxl, 'The maximal factorizations of the finite simple groups and their automorphism groups', Mem. Amer. Math. Soc. No. 432 (1990), 17-23.

[5] D. Meier and James Wiegold, 'Growth sequences of finite groups V', J. Austral. Math. Soc. (Series A) 31 (1981), 374-375.

[6] A. G. R. Stewart and James Wiegold, 'Growth sequences of fintely generated groups II', Bull. Austral. Math. Soc. 40 (1989), 323-329.

[7] J. Wiegold, 'Growth sequences of finite groups', J. Austral. Math. Soc. 17 (1974), 133-141.

[8] _ _ 'Growth sequences of finite groups II', J. Austral. Math. Soc. (Series A) 20 (1975), 225-229.

[9] ___, 'Growth sequences of finite groups III', J. Austral. Math. Soc. (Series A) 25 (1978), $142-144$.

[10] —_, 'Growth sequences of finite groups IV', J. Austral. Math. Soc. (Series A) 29 (1980), 14-16.

[11] James Wiegold and J. S. Wilson, 'Growth sequences of finitely generated groups', Arch. Math. 30 (1978), 337-343.

No. 31, 25th Edalat

Ahamd Abab Ave

Mashhad

Iran 